\title{
Physiotherapy in Urinary Dysfunction Post-Surgery for Endometriosis: Case Report
}

\author{
Authors: \\ Natália Mainardi Simas, ${ }^{1}$ Domingos Mantelli Borges Filho, ${ }^{2-6}$ Erica M. \\ C. Mantelli Borges, ${ }^{6,7}{ }^{*}$ Carla Maria de Abreu Pereira, ${ }^{1,6}$ Vera Lúcia dos \\ Santos Alves ${ }^{1,8,9}$ \\ 1. Santa Casa of São Paulo Medical Science College, São Paulo, Brazil \\ 2. Fetus Institute of Ultrasonography, São Paulo, Brazil \\ 3. USP Medical School, São Paulo, Brazil \\ 4. Scientific Technical Police of the State of São Paulo, São Paulo, Brazil \\ 5. Civil Police Academy of the State of São Paulo, São Paulo, Brazil \\ 6. Mantelli Clinic, São Paulo, Brazil \\ 7. Gynecology and Human Sexuality Sector of the Mantelli Clinic, São Paulo, Brazil \\ 8. Mogi das Cruzes University, São Paulo, Brazil \\ 9. Cardiopulmonary and Metabolic Physical Therapy Service of Santa Casa de São \\ Paulo, São Paulo, Brazil \\ *Correspondence to fisiocarlapereira@gmail.com
}

Disclosure:

Acknowledgements:

Received:

Accepted:

Keywords:

Citation:
The authors have declared no conflicts of interest.

Images were obtained from the Pelvic Physiotherapy Outpatient Clinic of the Irmandade da Santa Casa de Misericórdia de São Paulo, São Paulo, Brazil. The patient provided written, informed consent for the use of their photographs and case details for scientific study.

23.06.20

30.09 .20

Endometriosis, physical therapy, urinary retention, vulvar oedema.

EMJ Repro Health. 2021;DOI/10.33590/emjreprohealth/19-00133.

\section{Abstract}

Endometriosis is characterised by the presence of endometrial tissue outside the uterine cavity that responds to oestrogen and stimulates local inflammatory processes, adhesions, pelvic pain, and infertility. The treatment of endometriosis includes the use of medications and videolaparoscopy for excision of adhesions or lesions. Some complications are associated with the videolaparoscopy, such as vascular, intestinal, urinary, neurological, and more rarely, vulvar oedema, which makes the rehabilitation difficult in the immediate postoperative period. In relation to the urinary dysfunction and to the vulvar oedema, physiotherapy has resources with demonstrated efficacy in the treatment of such complications after videolaparoscopy; they can rehabilitate the patient and improve their quality of life in a short time. In this study, the authors report the case of a patient treated by the Physical Therapy Service of the Santa Casa de Misericórdia Hospital of São Paulo, São Paulo, Brazil, with urinary retention and vulvar oedema after videolaparoscopy for endometriosis, which showed improvement in vulvar oedema with the application of physiotherapy. 


\section{BACKGROUND}

Endometriosis is a chronic condition, characterised by the presence of endometrial tissue outside of the uterine cavity, such as the pelvic cavity, the rectum, the ovary, and the uterosacral ligament and in rare cases also in the diaphragm, pleura, and pericardium. Endometriosis responds to oestrogen hormone stimulation, which stimulates local inflammatory processes, accompanied by adhesions, fibrosis, neuronal infiltration, and anatomical distortion, which contribute to the presence of pelvic pain and infertility. 1-3 $^{-3}$

Endometriosis affects $6-10 \%$ of females of reproductive age and is seen in up to $60 \%$ of those who present with pelvic pain complaint and in up to $50 \%$ of cases of infertility. ${ }^{3}$ The diagnosis is based on the clinical history of the patient, signs and symptoms, physical examination, medical imaging techniques (e.g., MRI), and proven by histological examination of the materials collected during the laparoscopy. ${ }^{4}$ Several theories attempt to explain the pathogenesis of the condition and can be divided into those that suggest that the implants originate from endometrial tissue, such as the retrograde menstruation theory, and those that propose a nonuterine origin, such as the theory of coelomic metaplasia. ${ }^{5,6}$

According to the American Society for Reproductive Medicine (ASRM), endometriosis can be classified into four stages (mild to severe), with different degrees of impairment and affected areas in each of them. ${ }^{7}$ The treatment for endometriosis includes oral progestogen contraceptives, testosteronederived agonists of gonadotropin releasing hormone, and nonsteroidal anti-inflammatory drugs. $^{8}$ Surgery plays an important role in the control of endometriosis and aims to completely ablate and/or excise the endometrial tissue and to correct anatomical changes caused by adhesions or lesions. ${ }^{9}$ Laparoscopy is considered to be the gold standard test for the diagnosis of this pathology and resection of endometriosis through this technique is effective in pain control. ${ }^{10}$ Vulvar oedema and urinary retention are some of the urinary complications related to videolaparoscopy. ${ }^{9}$

Postlaparoscopic vulvar oedema is a rare complication, but when presented it may affect the patient's urination. Unfortunately, there are still no guidelines for its management and the physiopathology associated with the procedure is unknown.. ${ }^{11}$ The studies of Guven et al. ${ }^{11}$ and of Trout et al. ${ }^{12}$ reported cases of patients who underwent laparoscopic surgery and presented with vulvar oedema in the postoperative period. Consequently, urination was impaired and relief catheterisation to decrease the oedema and rest were required. The authors observed that the decreasing of oedema was associated with an improvement in diuresis. It is important to mention that vulvar oedema can also have other causes, such as vulvar trauma as a result of uterine manipulation during hysterectomy and voiding dysfunction in the postoperative period of females with deep endometriosis undergoing surgery can be secondary to other causes (neurogenic dysfunctions of the pelvic floor).

\section{CASE REPORT}

Described here is the physiotherapeutic intervention in vulvar oedema and urinary retention presented in the postoperative period of videolaparoscopy for the treatment of endometriosis.

The case report followed the sequence of identification, main complaint, history of the current disease, and evolution. The research instruments used in this case were anamnesis, evaluation of the pelvic floor with visual inspection, and physiotherapeutic care (seven sessions).

A 45-year-old female of Caucasian background was diagnosed with endometriosis, on the 3rd postoperative day of right oophoroplasty with hysterectomy and bilateral salpingectomy and right pararectal tumour excision. Following removal of the bladder catheter, the patient developed urinary retention and urinary incontinence as a result of overflow; the catheter was inserted again and maintained. The patient was evaluated in bed by the Pelvic Physiotherapy Service of the Irmandade da Santa Casa de Misericórdia de São Paulo on the fifth postoperative day. In the inspection, vulvar oedema and altered sensitivity of the external region of the vulva were observed (Figure 1A). Sensitivity in the vulva region was assessed by the Semmes-Weinstein Monofilament (SWM) test. The patient was instructed by the 
physiotherapy team to apply cryotherapy (ice pack) to the vulvar region for 20 minutes and perform stimulation in the inguinal lymph nodes (20 circles in the inguinal lymph node chain), twice a day.

The patient was discharged on the 5th postoperative day and returned to the physiotherapy outpatient clinic after 48 hours for the first session of physical therapy, already showing improvement of the oedema (Figure 1B).

During the first postdischarge session, functional electrical stimulation was performed in the supra pubic region and in the labia majora (Figure 2), with a pulse width of 500 microseconds, 50 $\mathrm{Hz}$ frequency, 2-second ramp rise, 5-second maintenance, 2-second ramp descent, with an application time of 10 minutes. . $^{13,14}$ A device with two channels was used: adhesive electrodes $(5 \mathrm{~cm} \times 5 \mathrm{~cm}$ ) were applied in the supra pubic region and adhesive electrodes $(3 \mathrm{~cm} \times 5$ $\mathrm{cm}$ ) in the labia majora; the application was simultaneous. The purpose of electrostimulation was to activate muscle contraction to perform a mechanism of pumping the accumulated liquids in the application regions. ${ }^{13,14}$

Transcutaneous electrical nerve stimulation current was also applied in the presacral region (S2 to S4) for neuromodulation, with a pulse width of 250 microseconds and a frequency of $8 \mathrm{~Hz}$ for 30 minutes, aiming to improve urinary retention. The patient was instructed to perform perineal exercises 3 times a day (the patient was in the supine position, with abducted hips, bent knees, and feet on the bed). The exercise consisted of contracting the pelvic floor for 5 seconds and relaxing it for 10 seconds, for 5 times. The patient was also instructed to maintain the application of cryotherapy to the vulvar region as well as the stimulation of the inguinal lymph nodes (20 circular movements).

During the second physiotherapy session, performed in the same week, the patient was no longer using a bladder catheter, the oedema had decreased, and they reported a sensation of incomplete emptying of the bladder (Figure 3). The conduct was maintained, and the patient was instructed to perform scheduled urination every 3 hours.

At the third session, the patient no longer presented with oedema, they reported a strong urinary stream, good urine flow, and postvoid residual; the conduct was maintained until the sixth session.

\section{GENERAL CONSIDERATIONS}

Operative laparoscopy has undergone technological advances in the last decade." According to several studies, vulvar oedema is a complication that rarely occurs in the postoperative period of laparoscopy, which may explain the lack of studies on the subject.11,12,15,16 The first description of this complication, related to laparoscopic surgery, was made by Trout and Kemmann in 1996.12 The researchers reported a case of three patients undergoing laparoscopy for treatment of ovarian cyst, pelvic adhesions, and gamete intrafallopian transfer, who presented with vulvar oedema in a 24-hour postoperative period, as well as discomfort and inability to urinate. The patients were hospitalised and received traditional treatment with Foley catheter for urine drainage, local application of ice, and rest. Their condition improved between 1 and 3 days, but one patient developed a urinary tract infection. The researchers also reported that in their 3 years' of experience, they only observed three cases of vulvar oedema among approximately 900 laparoscopies, which confirms the low rate of occurrence of this complication. The studies of Pados et al..$^{15}$ and Guven et al. ${ }^{11}$ reported vulvar oedema in laparoscopic cystectomy. In the report by Pados et al., the patient presented with discomfort and vulvar oedema 3 hours after the surgery. The treatment consisted of a Foley catheter, introduced to prevent urinary retention due to the worsening of their condition; application of ice to the vulva; rest for the patient; and bandaging of the oedema after application of topical steroid cream. The oedema improved after a few minutes and the catheter and the bandage were removed after 48 hours and the patient was discharged from hospital. In the report by Guven et al., the patient was readmitted 27 hours after the procedure with vulvar oedema and inability to urinate. In parallel to the study by Pados et al., the treatment was performed with application of ice to the vulva, Foley catheter, and rest, with improvement of the oedema in 30 hours and hospital discharge after 2 days, without complications. These results are similar to the case report presented here. 

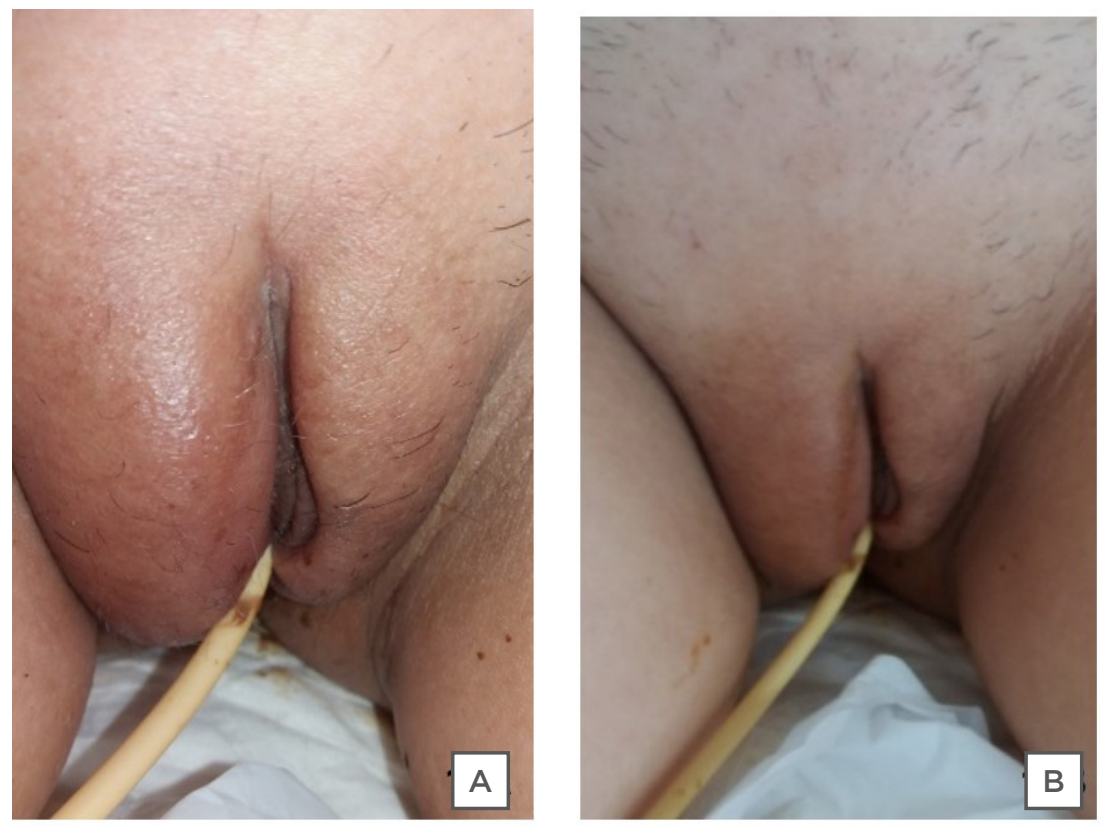

Figure 1: Postoperative vulvar oedema.

A) Vulvar oedema at the time of evaluation of physical therapy on the 5th postoperative day. B) Improvement of the oedema and of urination after 48 hours of hospital discharge.

Source: Pelvic Physiotherapy Outpatient Clinic of the ISCMSP (Irmandade da Santa Casa de Misericórdia de São Paulo).

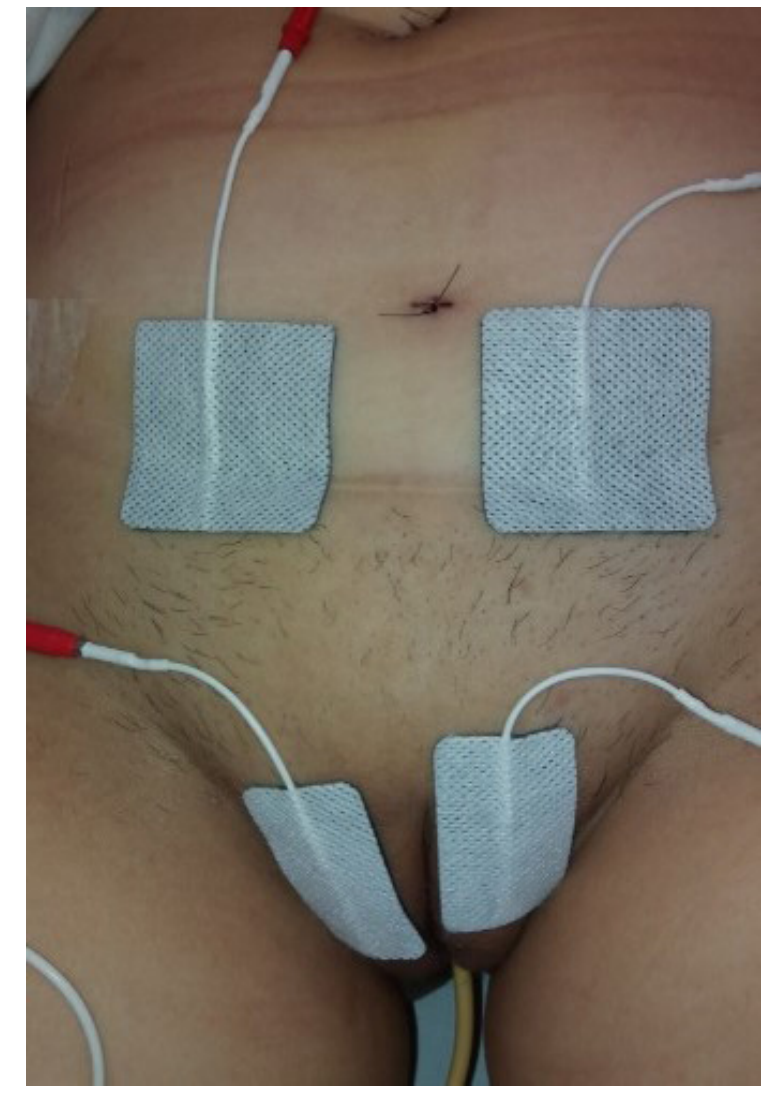

Figure 2: Surface electrostimulation. 

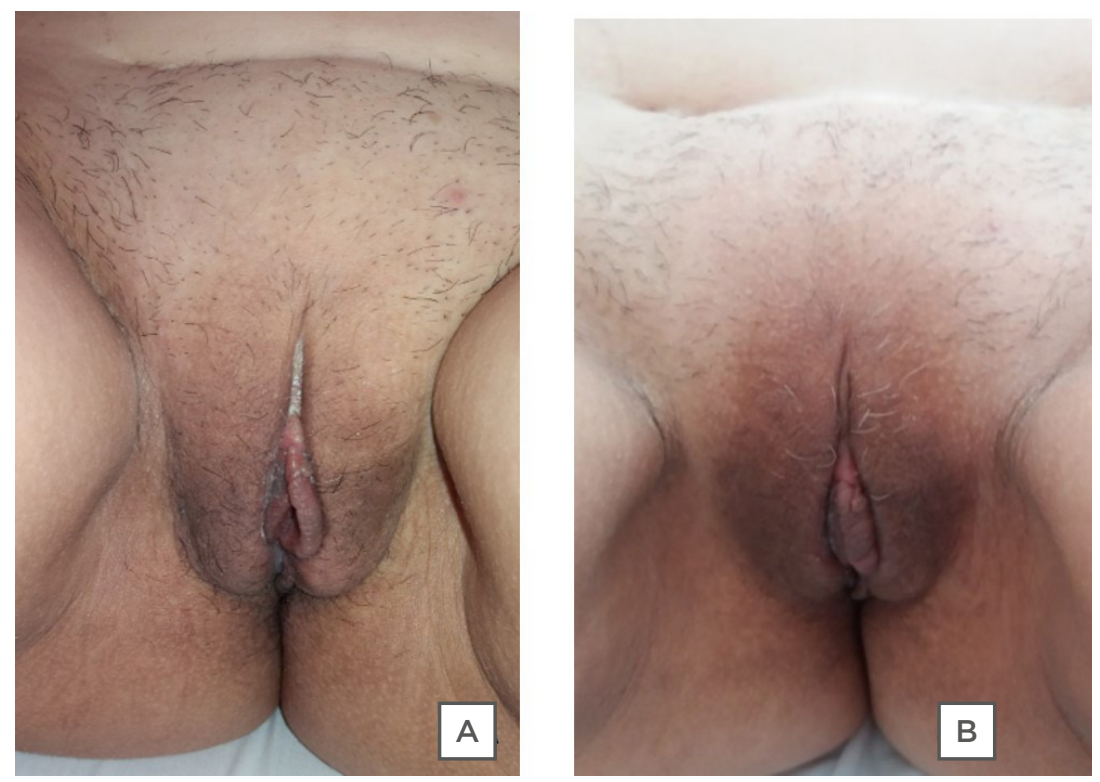

Figure 3: Decreased vulvar oedema during physiotherapy treatment.

A) During the second session of physiotherapy, the patient did not require the use of a bladder catheter and showed improvement of the oedema and urinary retention. B) During the seventh session, the patient had no complaints and no urinary retention; they were discharged from physiotherapy treatment.

It was possible to observe that the time of onset of vulvar oedema in the postoperative period and the time elapsed for its improvement varied in all the studies. A common complication was urinary retention, a consequence of vulvar oedema, which makes it impossible for the urine to be released as the oedema compresses the urethra. Its cause is complex and unpredictable, and its incidence rate associated with laparoscopy varies between $1.2 \%$ and $22.9 \% .^{16}$ The pathophysiology of vulvar oedema is still unknown." One of the hypotheses is the intraoperative or postsurgery migration of an intra-abdominal fluid from a suprapubic puncture area to the vulvar subcutaneous tissue.15,16 The persistence of the canal of Nuck may also be one of the causes of fluid leakage from the peritoneal cavity to the vulvar region. ${ }^{11,15,17,18}$ The canal of Nuck is a small wrapping of the parietal peritoneum, it is connected to the uterus by the round ligament through the inner inguinal ring in the inguinal canal and it communicates with the labia majora. It is a predelivery canal, which is closed at birth or disappears in the first year of life. When this communication remains open it may be the cause of the inguinal hernia and of the hydrocele of the canal of Nuck. ${ }^{18,19}$ Reed and Robinson ${ }^{18}$ related the use of $4 \%$ icodextrin in laparoscopic gynaecological surgery to vulvar oedema and urinary retention presented by one patient. Icodextrin is used laparoscopically at the time of surgery to prevent the formation of adhesions that can lead to complications such as infertility, chronic pelvic pain, and small intestine obstruction. The authors reported the case of a patient readmitted after laparoscopic salpingectomy using $4 \%$ of icodextrin to prevent adhesions. The patient complained of inability to urinate and presented significant vulvar oedema on physical examination. According to the authors, few complications are reported in the literature associated with the use of this substance, and the mechanism of vulvar oedema associated with it is unknown. However, they considered the patient's persistence of the canal of Nuck as a possible cause of vulvar oedema, since the substance is expected to remain in the peritoneal cavity until it is absorbed by the lymphatic system.

Regarding the management of vulvar oedema, it was observed that cryotherapy was a common therapeutic resource in the case reports discussed here, possibly because it presents results in the control and treatment of acute oedema with solid physiological and well-established bases in the literature, in addition the fact that it is a resource of low cost and easy application. ${ }^{20,21}$ The intervention in the management of vulvar oedema the authors applied was composed, in addition 
to cryotherapy, of electrostimulation, perineal exercises, and inguinal ganglion stimulation, which together presented excellent results. However, citations about the application of these resources in the other reports presented here were not found in author publications. The authors also did not comment if physical therapy was performed at some point during the treatment of the patients. Pinto e Silva et al. ${ }^{22}$ reported cases of four patients with vulvar oedema due to different aetiologies: one case of cervical cancer, one case of bilateral adrenalectomy postoperative for pheochromocytoma, and two cases of pregnancy with pre-eclampsia. The physical therapy treatment consisted of manual lymphatic drainage in the vulva region; stimulating lymphatic flow towards the inguinal lymph nodes; multilayer compression therapy, with overlapping bandages throughout the genital area, arranged as an underwear, aiming at lymphatic reabsorption and stimulation of lymphatic transport; and skin care. The physiotherapists used their knowledge of applying compressive bandages to upper and lower limbs to adapt the technique to the genital area. The researchers observed that this intervention had faster results in the resolution of vulvar oedema (mean: 3.5 days) compared with other studies in which the oedema was not treated, or other types of intervention were used.

\section{CONCLUSION}

The physiotherapeutic performance presents useful knowledge and resources to assist in the treatment of vulvar oedema and urinary retention. These complications are rare, but they may occur in the postoperative period for the treatment of endometriosis. The importance of physical therapy practise in these complications should be emphasised. Although endometriosis has no cure, it can be treated and controlled through periodic follow-up.

\section{References}

1. Acién P, Velasco I. Endometriosis: a disease that remains enigmatic. ISRN Obstet Gynecol. 2013; http://dx.doi. org/10.1155/2013/242149.

2. Maggiore ULR et al. A systematic review on endometriosis during pregnancy: diag-nosis, misdiagnosis, complications and outcomes. Hum Reprod Update. 2016;22(1):70-103.

3. Giudice LC. Clinical practice: endometriosis. N Engl J Med. 2010;362(25):2389-98.

4. Dunselman GAJ et al. ESHRE guideline: management of women with endometrio-sis. Hum Reprod 2014;29(3):400-12.

5. Burney RO, Giudice LC. Pathogenesis and pathophysiology of endometriosis. Fertil Steril. 2012;98(3):511-9.

6. Marques AAM, Petta CA, "Fisioterapia na endometriose," Tratado De Fisioterapia Em Saúde Da Mulher (2011), São Paulo: Roca, pp.345-50. [In Portuguese].

7. American Society for Reproductive Medicine (ASRM). Revised American Society for Reproductive Medicine classification of endometriosis: 1996. Fertil Steril. 1997;67(5):817-21.

8. Abrão MS, Gaec SP, "Tratamento da endometriose por laparoscopia operatória," Pi-notti JA et al. (eds.), Tratado de Ginecologia: Condutas e Rotinas da Disciplina de Ginecologia da Faculdade de Medicina da Universidade de São Paulo - USP
(2005), Rio de Janeiro: Revinter pp.562-6. [In Portuguese].

9. Neme RM et al. "Complicações em laparoscopia," Pinotti JA et al. (eds.), Tratado de Ginecologia: Condutas e Rotinas da Disciplina de Ginecologia da Faculdade de Me-dicina da Universidade de São Paulo - USP (2005), Rio de Janeiro: Revinter, pp.562-6. [In Portuguese].

10. Kondo $\mathrm{W}$ et al. Tratamento cirúrgico da endometriose baseado em evidências/ evi-dence-based surgical treatment of endometriosis. Femina. 2011;39(3):143-8.

11. Guven S et al. Vulvar edema as a rare complication of laparoscopy. J Am Assoc Gy-necol Laparosc. 2004;11(3):429-32.

12. Trout SW, Kemmann E. Vulvar edema as a complication of laparoscopic surgery. J Am Assoc Gynecol Laparosc. 1996;4(1):81-3.

13. Hwang UJ et al. Pelvic floor muscle parameters affect sexual function after 8 weeks of transcutaneous electrical stimulation in women with stress urinary incontinence. Sex Med. 2019;7(4):505-13.

14. Vallinga MS et al. Transcutaneous electrical nerve stimulation as an additional treatment for women suffering from therapy-resistant provoked vestibulodynia: a feasibility study. J Sex Med. 2015;12(1):228-37.

15. Pados $\mathrm{G}$ et al. Unilateral vulvar edema after operative laparoscopy: a case report and literature review. Fertil Steril. 2005;83(2):471-3.

16. Nesbitt-Hawes EM et al. Urinary retention following laparoscopic gynaecological surgery with or without 4\% icodextrin anti-adhesion solution. Aust N Z J Obstet and Gynaecol. 2013;53:305-9.

17. Bhairavi S et al. Postparacentesis vulvar edema in ovarian hyper stimulation syn-drome. Int J Reprod Contracept Obstet Gynecol. 2016;5(11):4064-6.

18. Reed B, Robinson R. Postoperative urinary retention with gross vulvar edema after use of $4 \%$ icodextrin. Mil Med. 2015;180(7):e858-60.

19. Amani MR. Hernie inguinale chez la fille. 2018. Available at: http://www. chirurgie-pediatrique.net/pathologiede-paroi-/hernie-inguinale-chez-lafille/. Last ac-cessed: 01 February 2021. [In French].

20. Kwang SK et al. Hydrocele of the canal of nuck in a female adult. Arch Plast Surg. 2016;43(5):476-78.

21. Guirro R et al. [The physiological effects of cryotherapy: a review] Rev. Fisiot. 1999;6(2):164-70. [In Portuguese].

22. Pinto e Silva MPP et al. Manual lymphatic drainage and multilayer compression therapy for vulvar edema: a case series. Physiother Theory Pract. 2015;31(7):527-31. 